\title{
Bubbles and Superbubbles: Observations and Theory
}

\author{
You-Hua Chu \\ Astronomy Department, University of Illinois, 1002 W. Green Street, Urbana, IL 61801, USA \\ email: chu@astro.uiuc.edu
}

\begin{abstract}
Massive stars inject energy into the surrounding medium and form shell structures. Bubbles are blown by fast stellar winds from individual massive stars, while superbubbles are blown by fast stellar winds and supernova explosions from groups of massive stars. Bubbles and superbubbles share a similar overall structure: a swept-up dense shell with an interior filled by low-density hot gas. Physical properties of a bubble/superbubble can be affected by magnetic field, thermal conduction, turbulent mixing, inhomogeneous ambient medium, etc. I will review recent progresses on observations and compare them to theoretical expectations for (1) swept-up dense shells, (2) hot interiors, and (3) interface between a dense shell and its interior hot gas
\end{abstract}

Keywords. ISM: bubbles - ISM: structure - circumstellar matter - stars: mass loss - stars: Wolf-Rayet - stars: winds, outflows - supernova remnants

\section{Introduction - Bubbles, Superbubbles, and Supergiant Shells}

Massive stars inject energy into the interstellar medium (ISM) via UV radiation, fast stellar wind, and ultimately supernova explosion. Depending on the concentration and evolutionary status of the massive stars, these energetic interactions produce different shell structures with sizes ranging from fractions of $1 \mathrm{pc}$ to greater than 1,000 pc. These shells are commonly detected in the $\mathrm{H} \alpha$ line, but the large shells without ionizing fluxes are detected only in the H I 21-cm line.

The hierarchy of interstellar shells is best illustrated by the supergiant shell (SGS) LMC-4. SGSs with diameters of $\sim 1000$ pc are the largest interstellar structures in a galaxy. Nine SGSs in the Large Magellanic Cloud (LMC) have been identified using $\mathrm{H} \alpha$ images (Goudis \& Meaburn 1978; Meaburn 1980), but they do not have a one-to-one correspondence with the SGSs identified from H I 21-cm maps (Kim et al. 1999) because these SGSs have different star formation histories and available ionizing fluxes (Book et al. 2008). LMC-4 is the largest SGS in the LMC, and as shown in Figure 1 its overall $\mathrm{H} \alpha$ emission originates from the ionized inner wall of its $\mathrm{H}$ I shell. The periphery of LMC4 is dotted with prominent H II regions, superbubbles, supernova remnants (SNRs), and bubbles. While the SGS LMC-4 stretches over 1,000-1,500 pc, the superbubbles N51D and N57A on the south rim of LMC-4 are 100-200 pc across, and the bubble N57C is only $\sim 25$ pc in size.

SGSs, superbubbles, and bubbles are three distinct classes of objects powered by massive stars. SGSs have sizes $\sim 10^{3} \mathrm{pc}$, dynamic ages of $\sim 10^{7} \mathrm{yr}$, and require multiple generations or episodes of star formation; for example, LMC-4 contains older OB associations in its interior and younger associations along its periphery (Book et al. 2008). Superbubbles have sizes $\sim 10^{2} \mathrm{pc}$, dynamic ages of $\sim 10^{6} \mathrm{yr}$, and require only one episode of star formation; for example, the OB associations LH 54 and LH 76 are responsible for creating the superbubbles N51D and N57A, respectively (Braunsfurth \& Feizinger 1983; Oey \& Smedley 1998). Bubbles have sizes up to a few $\times 10 \mathrm{pc}$, and are powered by the 


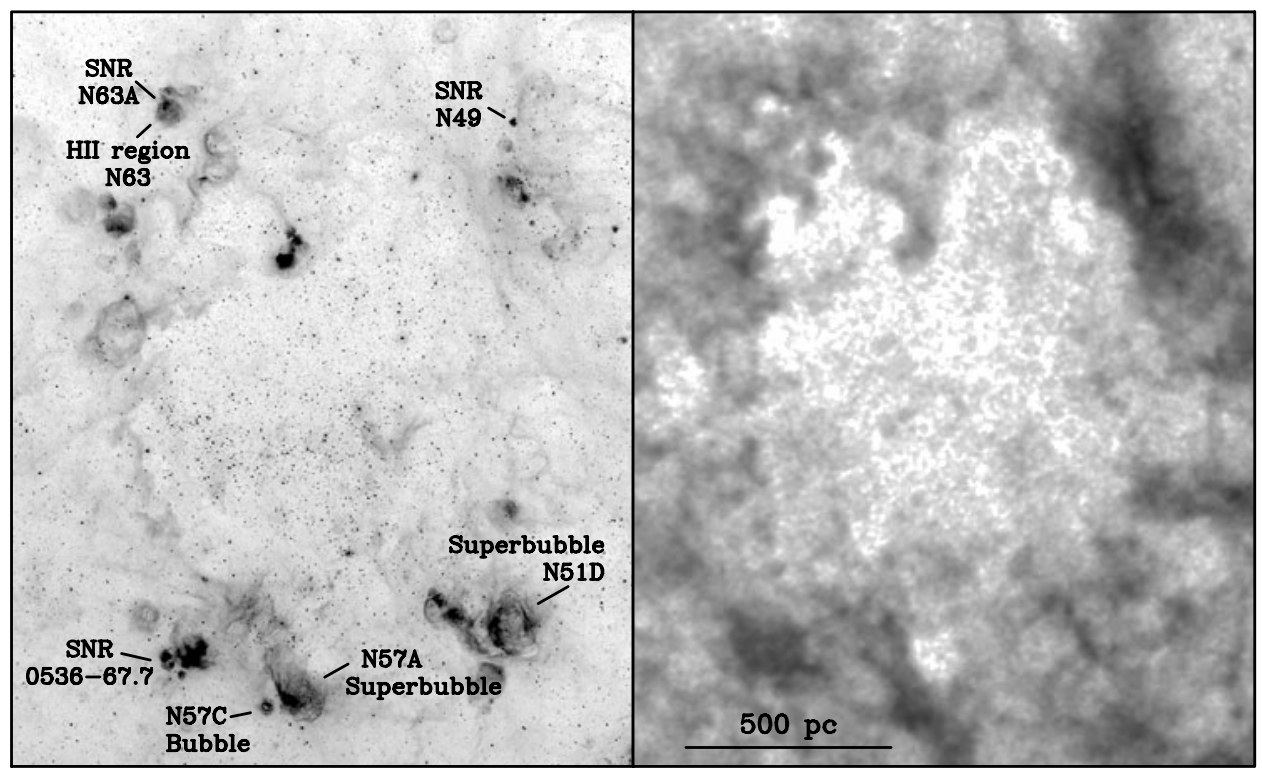

Figure 1. H $\alpha$ (left) and H I 21-cm (right) images of the supergiant shell LMC-4. The H $\alpha$ image of LMC-4 shows recent star formation activity along its rim, and the stellar energy feedback has produced superbubbles and bubbles, as well as prominent H II regions and supernova remnants. This image nicely illustrates the relative sizes of supergiant shell, superbubble, and bubble.

stellar wind of individual massive stars; for example, the bubble N57C is blown by the Wolf-Rayet (WR) star HDE 269748 (Chu \& Lasker 1980).

Bubbles blown by individual massive stars evolve along with the central stars. During the main sequence phase, a massive star is surrounded by the ISM and its fast stellar wind blows an interstellar bubble. As the massive star evolves into the red supergiant (RSG) or luminous blue variable (LBV) phase, the copious mass loss forms a small circumstellar nebula inside the central cavity of the main sequence bubble. As the massive star evolves further into a WR phase, the fast stellar wind sweeps up the circumstellar material and forms a circumstellar bubble. The evolution of nebulae around massive stars has been modeled by, e.g., García-Segura et al. (1996a,b) and Freyer et al. (2003, 2006), and observations of such nebulae throughout the HR diagram were reviewed by Chu (2003).

\section{Theories of Bubbles and Superbubbles}

Theories of wind-blown bubbles were initiated in the late 60's (e.g., Mathews 1966; Pikel'ner 1968; Pilkel'ner \& Shcheglov 1969), but the term "bubble" was not coined until the mid 70's (Castor et al. 1975). Bubbles were modeled assuming "momentum conservation" in which case fast stellar wind impinges on the bubble shell directly and imparts the out-going momentum (Steigman et al. 1975), or "energy conservation" in which case fast stellar wind is adiabatically shocked to high temperature and the thermal pressure of the hot gas drives the expansion of of the bubble shell (Dyson \& de Vries 1972; Castor et al. 1975).

The first and most comprehensive model of interstellar bubbles that aimed at explaining UV and X-ray observations was presented by Weaver et al. (1977). This model assumed a homogeneous ambient medium that might not be realistic, but it included essential physical processes and became a seminal paper, a true classic. The physical 

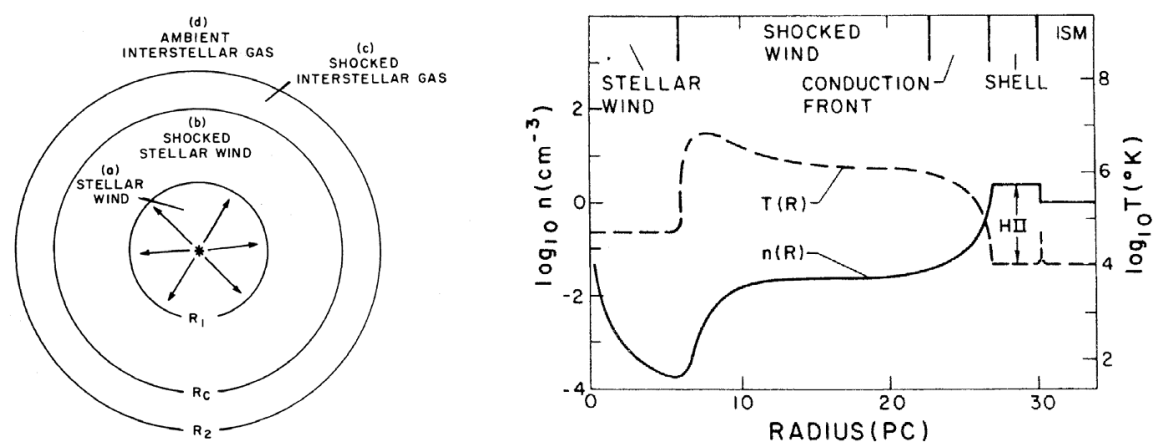

Figure 2. Left - A schematic drawing of the structure of an interstellar bubble. Right - temperature and density profiles of a pressure-driven interstellar bubble. Both figures are adopted from Weaver et al. (1977).

structure of a bubble is schematically shown in Figure 1 of Weaver et al. (1977) and reproduced in the left panel of Figure 2. At the center of a bubble, zone (a), the fast stellar wind expands freely from a massive star. It encounters an adiabatic stagnation shock at radius $R_{1}$, and the shocked stellar wind accumulates in zone (b). In the outer parts of a bubble, the ambient interstellar gas in zone (d) is shocked by the expanding bubble at radius $R_{2}$, and the shocked interstellar gas accumulates in zone (c). The shocked stellar wind and the shocked interstellar gas are separated by a contact discontinuity at radius $R_{c}$. The temperature and density profiles of Weaver et al.'s bubble are reproduced in the right panel of Figure 2. These profiles qualitatively manifest three physical actions. First, the stellar wind shock at $R_{1}$ is adiabatic, because the wind has high velocity and low density and the cooling time scale of the post-shock gas is long. Second, the interstellar shock at $R_{2}$ is isothermal because the interstellar gas is dense and the post-shock gas cools rapidly. Third, thermal conduction takes place at $R_{c}$, the interface between the shocked stellar wind and the shocked interstellar gas.

Weaver et al. (1977) model has formed the basis of numerous bubble models with more complex conditions in the ambient medium and time-dependent stellar winds. GarcíaSegura et al. (1996a,b) considered mass-loss rates and stellar wind velocities that varied along stellar evolution, and modeled the development of interstellar bubble during the main sequence and circumstellar bubble during the WR phase. Circumstellar bubbles are notably different from Weaver et al.'s interstellar bubble because of the $\propto r^{-2}$ density profile in the circumstellar medium. Instabilities in the dense swept-up circumstellar bubble shells cause fragmentation and clumpy morphologies. Freyer et al. $(2003,2006)$ furthered García-Segura et al. models by adding radiation effect; their radiative hydrodynamic simulations show that photoionization significantly influence the morphological evolution of interstellar bubbles formed during the main sequence stage. Arthur et al. (1993, 1996) and Pittard et al. (2001a,b) added another important physical process to bubble models - mass-loading by conductive evaporation or hydrodynamic ablation of cold clumpy material embedded in the hot bubble interior. The net effect of mass-loading is a faster cooling of the bubble interiors.

Superbubbles are powered initially by fast stellar winds and later by additional supernova explosions. For supernovae exploding in the low-density interior of a superbubble, the SNR shocks heat the already-hot surroundings; the shocks may be decelerated and become thermalized without ever reaching the dense swept-up shell. The effects of supernova explosions can thus be approximated by a stellar wind with a mechanical luminosity equal to the average supernova energy injection rate. Therefore, Weaver et al. model for 
interstellar bubbles has also formed the basis of superbubble models (e.g., Mac Low \& McCray 1988). Superbubbles modeled with the consideration of interstellar density gradient out of the galactic plane can produce blowouts into the galactic halo (Mac Low et al. 1989). The expansion and blowout of a superbubble can be impeded in the direction perpendicular to the magnetic field of the ISM (Tomisaka 1992).

\section{Observations Confronting Theories in the 80's and 90's}

Bubble models were inspired by optical observations of nebular dynamics and Copernicus observations of interstellar O VI absorption (Jenkins \& Meloy 1974). Advances in optical high-dispersion spectroscopic observations and NASA's UV and X-ray missions in the 80's and 90's made it possible to compare observations with models.

Optical observations can be used to determine the density, radius, and expansion velocity of the swept-up bubble shells. UV spectroscopic observations of massive stars can be used to determine stellar wind velocities and mass loss rates. If all of these observables are available for a bubble, it will be possible to critically test bubble models. It is found that circumstellar bubbles, most notably the well-observed NGC 6888, expand too slowly for their observed stellar wind velocity and mass loss rate, and nebular size and density (García-Segura et al. 1996a). The stellar wind mass loss rate can be revised down by a factor of a few because of the clumping of wind (Moffat \& Robert 1994), but the discrepancy between observations and model expectations is still significant.

Einstein X-ray observations detected diffuse emission from bubbles and superbubbles, but the data quality did not allow spectral analysis to determine plasma temperature and foreground absorption; assumptions of temperature and absorption had to be made in order to estimate X-ray luminosity. X-ray emission from the circumstellar bubble NGC 6888 was an order of magnitude lower than expected from models (Bochkarev 1988). A number of superbubbles in the LMC were also detected by Einstein, but their Xray luminosities were higher than expected; it was suggested that off-center supernova explosions were responsible for the X-ray emission (Chu \& Mac Low 1990).

ROSAT X-ray observations had a higher angular resolution and higher sensitivity for soft X-rays than Einstein. ROSAT PSPC observations detected diffuse X-ray emission from the circumstellar bubbles NGC 6888 and S 308. The X-ray emission from NGC 6888 shows a plasma temperature of $\sim 2 \times 10^{6} \mathrm{~K}$ and a luminosity of $\sim 1.6 \times 10^{34} \mathrm{erg} \mathrm{s}^{-1}$, confirming the Einstein estimate of luminosity (Wrigge et al. 1994). The ROSAT PSPC observation of S 308 was centered on the WR star HD 50896. The PSPC entrance window's support structure happened to contain a ring similar in size to S 308 , and thus occulted a significant fraction of diffuse X-ray emission from the shell rim. Diffuse Xray emission was convincingly detected in S 308, but the low X-ray surface brightness and low $\mathrm{S} / \mathrm{N}$ made the spectral analysis difficult and unreliable (Wrigge 1999). ROSAT observations of a number of other bubbles did not detect diffuse X-ray emission.

ROSAT PSPC observations detected diffuse X-ray emission from the Galactic superbubble in the Omega Nebula (M17). Spectral analysis shows the existence of plasma with temperatures as high as $\sim 8.5 \times 10^{6} \mathrm{~K}$ and an X-ray luminosity, $2.5 \times 10^{33} \mathrm{erg} \mathrm{s}^{-1}$, two orders of magnitude lower than expected, possibly caused by the strong magnetic field that hinders thermal conduction (Dunne et al. 2003). The OB association in M17 is so young that no supernova has occurred; the hot gas in M17 is likely powered by stellar winds solely. ROSAT PSPC observations have detected diffuse X-ray emission from the Eridanus superbubble, which is much larger and older than the M17 superbubble. The enhanced diffuse X-ray emission from the Eridanus superbubble, with a luminosity of 
$10^{35}-10^{36} \mathrm{erg} \mathrm{s}^{-1}$ and a plasma temperature of $\sim 2 \times 10^{6} \mathrm{~K}$, is likely caused by additional heating from a supernova explosion (Burrows et al. 1993).

ROSAT PSPC observations of $13 \mathrm{X}$-ray-bright superbubbles in the LMC show that their diffuse X-ray emission are more luminous than expected and are most likely energized by recent supernovae (Chu et al. 1993; Dunne et al. 2001). Deep ROSAT PSPC observations of three X-ray-faint LMC superbubbles yielded non-detections, and the $3 \sigma$ upper limits of their X-ray luminosities are similar to the expectations of models (Chu et al. 1995). Examinations of bubble dynamics indicate that X-ray-bright superbubbles expand faster than expected and X-ray-faint superbubbles roughly expand as expected from models, confirming the role played by supernovae (Oey 1996).

IUE and HST observations of UV absorption from conduction layers have been obtained in SiIV $\lambda \lambda 1393,1402$, C IV $\lambda \lambda 1548,1550$, and N V $\lambda \lambda 1238,1242$ lines. With the ionization potentials of Si III, C III, and N IV being 33.5, 47.0, and $77.5 \mathrm{eV}$, respectively, Si IV and C IV can be easily produced through photoionization by hot massive stars, but not $\mathrm{N} \mathrm{V}$, which needs to be produced through collisional ionization in a $10^{5} \mathrm{~K}$ medium, such as the conduction layer. As bubbles and superbubbles do encompass hot massive stars, the only reliable conduction layer indicator is the $\mathrm{NV}$ absorption, which is expected to be weak as the product of its abundance and oscillator strength is the lowest among the three species. IUE observations of interstellar absorption lines toward the central stars of NGC 6888 (Nichols-Bohlin \& Fesen 1993) and S 308 (Howarth \& Phillips 1986) show complex velocity structures in the CIV absorption lines with components corresponding to both the approaching side of the bubble shell and the foreground ISM. IUE observations of CIV and Si IV absorption from LMC superbubbles show mainly photoionized components from the superbubble shells, although some X-ray-bright superbubbles show velocity offsets between C IV absorption and $\mathrm{H} \alpha$ emission, possibly an influence of SNR shocks (Chu et al. 1994). The only convincing detection of conduction layer was provided by the HST GHRS observations of HD 50896 in S 308, based on the velocity structures of the C IV and N V lines (Boroson et al. 1997). Constrained by the Copernicus measurements of the O VI absorption, the observed N V absorption strength can be explained by a conduction front of Weaver et al. (1977) if the nitrogen abundance is enhanced by a factor of $\sim 10$, which is likely, as HD 50896 is a WN star.

In summary, observations of bubbles and superbubbles in the 80's and 90's have shown discrepancies from model expectations in bubble dynamics and X-ray luminosities. There is observational evidence of thermal conduction, but details are still uncertain.

\section{Astrophysical Observations of Bubbles and Superbubbles}

In 1999, the launch of Far UV Spectroscopic Explorer (FUSE), Chandra X-ray Observatory, and XMM-Newton X-ray Satellite made it possible to observe the hot interiors and conduction layers in bubbles and superbubbles with unprecedented resolution and accuracy so that bubble models can be critically examined. The launch of Spitzer Space Telescope in 2003 made it easy to identify massive young stellar objects so that their interstellar environments can be examined and used as realistic initial conditions for the formation of interstellar bubbles and superbubbles. In this section, I will review advances made in the 2000's for the three distinct layers of a bubble model: the swept-up shell, the hot interior, and the interface between them.

\subsection{Dense Swept-Up Shells}

It has been puzzling that Weaver et al. (1977) model of an interstellar bubble produces a dense swept-up shell, which should be ionized and visible in $\mathrm{H} \alpha$ images, but hardly 
any known main sequence $\mathrm{O}$ stars are surrounded by shell nebulae. The Bubble Nebula around the $\mathrm{O}$ star BD+60 2522 is an exception rather than the rule. In contrast, H I 21$\mathrm{cm}$ line observations frequently find expanding shells around evolved stars and the shell properties are consistent with those expected from interstellar bubbles (Cappa et al. 2003). Why aren't interstellar bubbles around main sequence O stars visible?

This puzzle is solved by the kinematically detected bubble shells in the LMC H II regions N11B and N180B (Nazé et al. 2001). Expanding shells exist around the O stars in these young H II regions, but their expansion velocities are only $15-20 \mathrm{~km} \mathrm{~s}^{-1}$. For a photoionized medium at $10^{4} \mathrm{~K}$, the isothermal sound velocity is $10 \mathrm{~km} \mathrm{~s}^{-1}$ and the observed shell expansion velocity drives only a weak shock into the ambient medium. Without a strong compression to produce sharp density contrast, these bubble shells cannot be identified morphologically over a complex background. If such a shell expands into a neutral medium that has an isothermal sound velocity of $\sim 1 \mathrm{~km} \mathrm{~s}^{-1}$, strong shocks and compression are generated, and the resultant large density contrast between the swept-up shell and the ambient medium makes the H I shell easily detectable.

The swept-up bubble shells in N11B and N180B have been compared with Weaver et al. (1977) interstellar bubble models. For the observed shell size, expansion velocity, and density, the required stellar wind mechanical luminosity is 1-2 orders of magnitude lower than that implied by the observed stellar content (Nazé et al. 2001). This problem is similar to that for the circumstellar bubble NGC 6888. While it is possible that stellar wind luminosity has been over-estimated, it is also possible that the interstellar environment is much more complex than a homogeneous medium assumed by Weaver et al. (1977). For example, the bubble structure in N44F ("Celestial Geode") shows dust pillars along the shell rim (Nazé et al. 2002); furthermore, massive young stellar objects have been identified in some dust pillars, indicating on-going star formation (Chen et al. 2008). For a bubble in a clumpy medium, the evaporation and ablation of dense clumps in the hot interior of a bubble raises the density and cooling rate, and thus may significantly change the bubble dynamics. It therefore may be over-simplistic to compare a bubble formed in a complex environment to a Weaver et al. bubble in a homogeneous medium.

H I surveys of the Milky Way and nearby galaxies have found large numbers of shells, e.g., 300 shells in the outer Milky Way (Ehlerová \& Palouš 2005), 500 shells in the Small Magellanic Cloud (Staveley-Smith et al. 1997), 23 supergiant shells and 103 giant shells in the LMC (Kim et al. 1999). These shells have a range of physical conditions in their ambient ISM, history of star formation, and evolutionary stages. Some of the "shells" may not be physical, especially those with sizes and expansion velocities comparable to the resolution limit of the H I observations. It is not clear whether physical insights can be unambiguously gained from the observed distributions of shell sizes or expansion velocities, although the size distribution of superbubbles in galaxies has been modeled by Oey \& Clarke (1997), assuming all shells have the same lifetime. A non-negligible fraction of the shells do not show underlying population of massive stars that are responsible for the formation of the shells (Hatzidimitriou et al. 2005). Deep observations of the stellar population within these shells to search for the massive stars' surviving lowermass siblings are needed before resorting to exotic origins,such as gamma-ray bursts.

\subsection{Hot Interiors}

Chandra observations of NGC 6888 and XMM-Newton observations of S 308 have yielded the highest-quality X-ray image and spectra of the hot interior gas on single-star bubbles. Figure 3 shows images of S 308 in [O III] $\lambda 5007$ and in X-ray. The X-ray image shows a strong limb-brightening, indicating that the emitting layer is thin. A close-up comparison between optical and X-ray images (Figure 4) shows a gap between the fronts of [O III] 


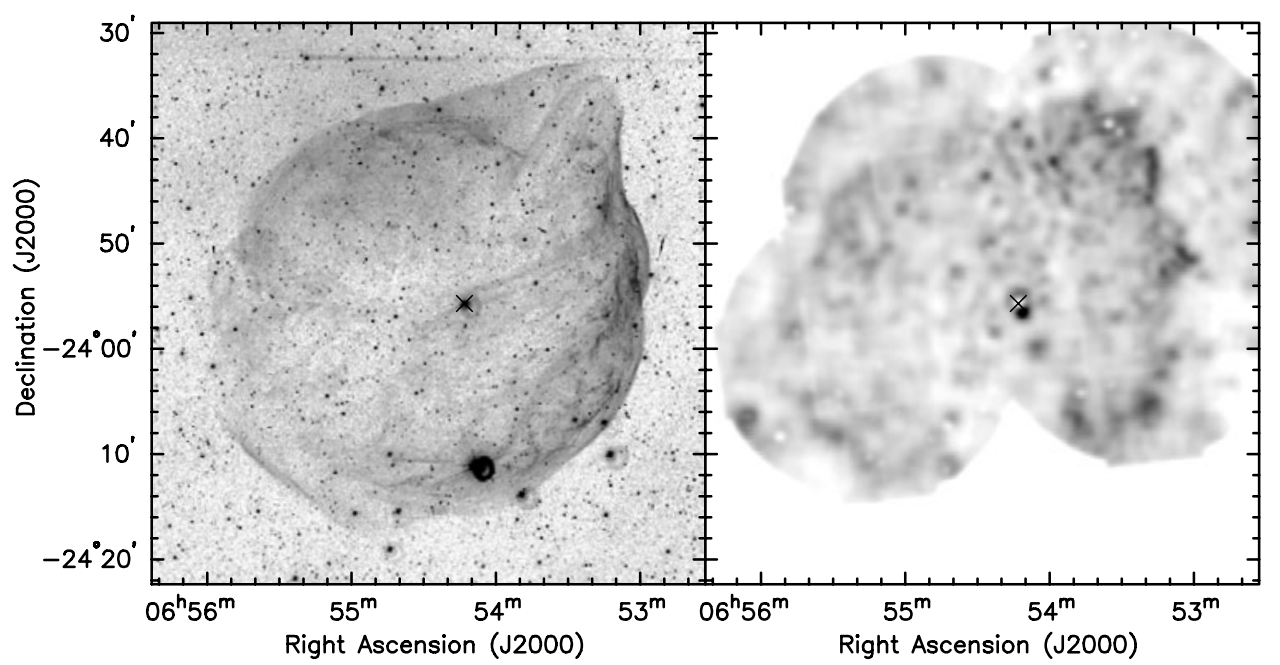

Figure 3. Left - [O III $] \lambda 5007$ image of S 308. Right - XMM-Newton EPIC-pn X-ray image of S 308 in $0.3-1 \mathrm{keV}$ band. Obvious point sources have been excised and adaptive smoothing has been applied. Diffuse X-ray emission inside S 308 shows a limb-brightened morphology. The central WN star HD 50896 is marked with a "X".
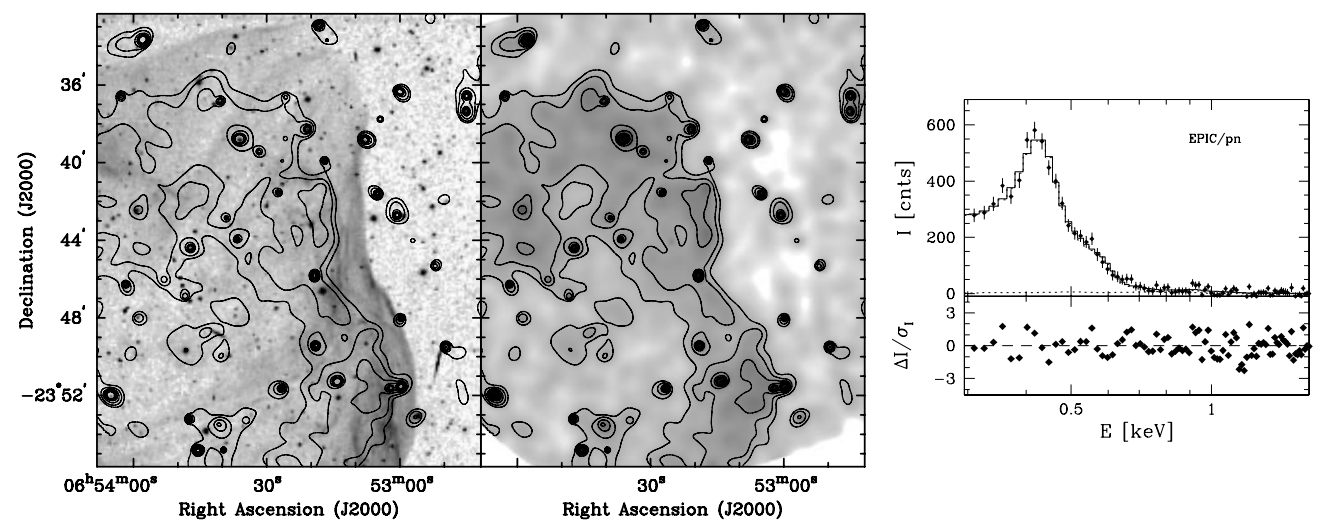

Figure 4. Left - [O III] and XMM-Newton EPIC/pn images of the NE quadrant of S 308 overlaid with X-ray contours. Point X-ray sources are not removed in this image; these point sources must have contributed to the X-ray emission above $1 \mathrm{keV}$ detected in the ROSAT PSPC data (Wrigge 1999). Right - XMM-Newton EPIC-pn spectrum of the diffuse emission in the NE quadrant of $\mathrm{S} 308$. These figures are from Chu et al. (2003).

and X-ray emission, and the X-ray spectrum shows that the X-ray emission is very soft, with hardly any emission above $1 \mathrm{keV}$ (Chu et al. 2003). Such soft X-ray emission is most susceptible to interstellar absorption. Indeed, S 308 is detectable because it is nearby, at $1.5 \pm 0.3 \mathrm{kpc}$, and has a small foreground absorption column density, $N_{\mathrm{H}} \sim 1 \times 10^{21} \mathrm{~cm}^{-2}$. The plasma temperature derived from spectral fits is $\sim 1.1 \times 10^{6} \mathrm{~K}$, and the rms electron density of the hot gas is $0.3-0.6 \mathrm{~cm}^{-3}$ (Chu et al. 2003).

Chu et al. (2003) compared X-ray and optical observations of S 308 with the analytical model of circumstellar bubbles by García-Segura \& Mac Low (1995), assuming that the optical shell expanding at $65 \mathrm{~km} \mathrm{~s}^{-1}$ consisted of swept-up RSG wind and that the expansion velocity of the RSG wind was $\leqslant 30 \mathrm{~km} \mathrm{~s}^{-1}$. They speculated that the gap between the X-ray emission edge and the optical emission edge might correspond to the 


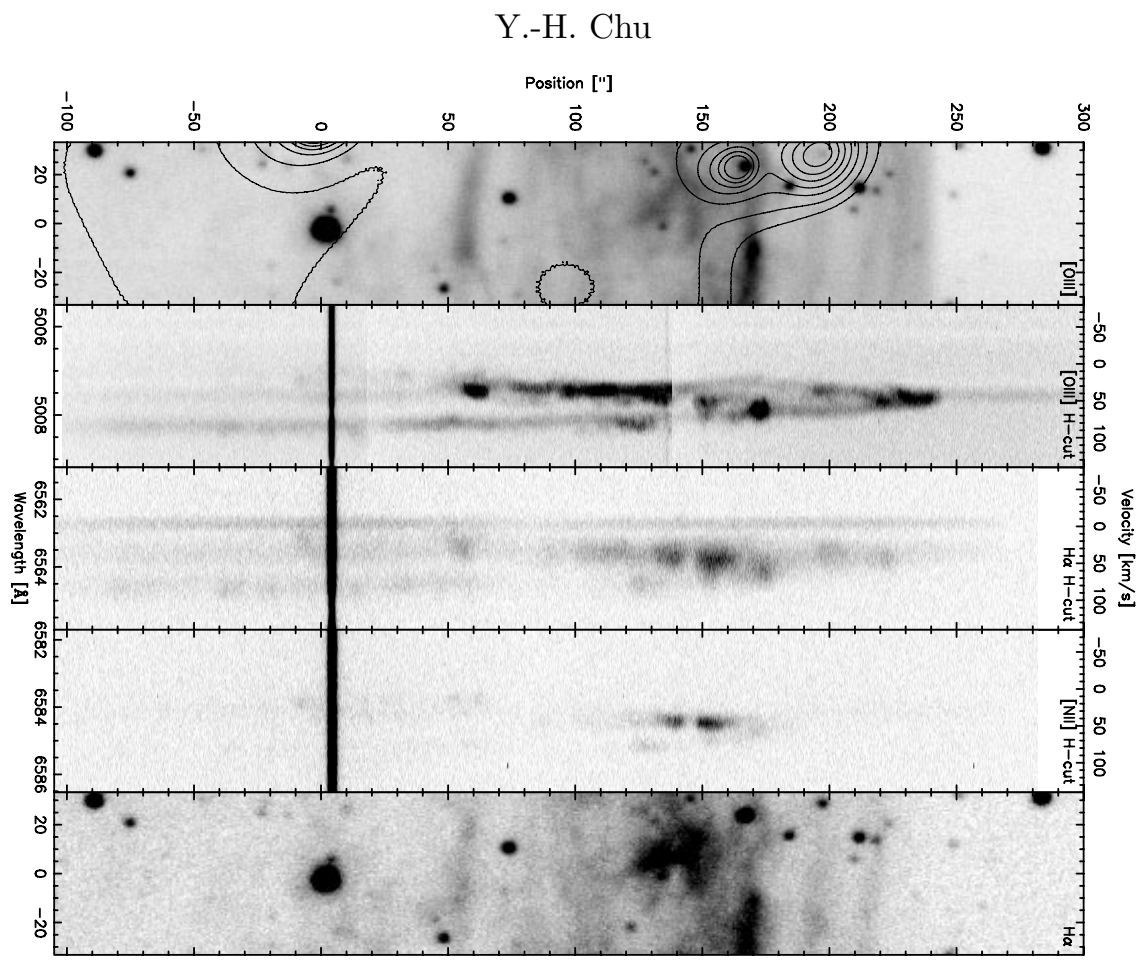

Figure 5. A narrow slice of the western shell rim of S 308. Top to bottom - [O III] image with X-ray contours; long-slit [O III] echellogram EW-oriented and passing through the star at the origin of the image above; $\mathrm{H} \alpha$ echellogram at the same position; [N II] echellogram at the same position; $\mathrm{H} \alpha+[\mathrm{N} \mathrm{II}]$ image of the same field shown in the [O III] image on top.

conduction layer. However, our recent high-dispersion long-slit echelle observations of the nebular shell of S 308 indicate a different interpretation. As shown in Figure 5, the "gap" between the [O III] front and the X-ray front has a "filled" line morphology in the [O III] echellogram. The continuous presence of emission at the systemic velocity across the gap indicates that material exists continuously along the radial direction perpendicular to the line of sight. Therefore, the "gap" between the [O III] front and the X-ray front is filled with nebular material. This filled layer most likely consists of RSG wind expanding at a uniform velocity of $65 \mathrm{~km} \mathrm{~s}^{-1}$. The bubble dynamics presented by Chu et al. (2003) therefore needs revision. Freyer et al. (2005) suggested that the diffuse X-ray emission originates from the swept-up RSG wind shell. The limb-brightening in both the direct images and the echelle line images in $\mathrm{H} \alpha$ and [O III] indicates the presence of a denser region that may correspond to the swept-up RSG wind shell. The low temperature and large mass of the X-ray-emitting material suggest that significant mixing of nebular material into the shocked fast wind has occurred.

Chandra observations of NGC 6888 reveal diffuse X-ray emission extending toward the optical shell rim, and the spectral fits confirm the plasma temperature of $\sim 2 \times 10^{6} \mathrm{~K}$ previously determined from ROSAT PSPC observations (Gruendl et al. 2003). Since a range of plasma temperatures is expected from the hot interior to the conduction front, the observed X-ray spectrum needs to be fitted by those calculated using bubble models (e.g., Strickland \& Stevens 1997).

Diffuse X-ray emission from Galactic H II regions, such as the Orion Nebula and the Rosette Nebula, were reported using Einstein observations, but ROSAT PSPC observations with higher resolution have resolved the diffuse emission into point sources. The high angular resolution and high sensitivity of Chandra and XMM-Newton make it possible to 
Table 1. Physical Properties of Hot Gas in Bubble Interiors

\begin{tabular}{cccc}
\hline Bubble Type & $\begin{array}{c}T_{\mathrm{e}} \\
{\left[10^{6} \mathrm{~K}\right]}\end{array}$ & $\begin{array}{c}N_{\mathrm{e}} \\
{\left[\mathrm{cm}^{-3}\right]}\end{array}$ & $\begin{array}{c}L_{\mathrm{X}} \\
{\left[\mathrm{erg} \mathrm{s}^{-1}\right]}\end{array}$ \\
\hline Orion Bubble & 2 & $0.2-0.5$ & $5 \times 10^{31}$ \\
WR Bubble & $1-2$ & 1 & $10^{33}-10^{34}$ \\
M17 Superbubble & $1.5,7$ & 0.3 & $3.4 \times 10^{33}$ \\
Planetary Nebula & $2-3$ & 100 & $10^{31}-10^{32}$ \\
\hline
\end{tabular}

separate point sources from faint diffuse emission. Diffuse X-ray emission from the Orion Nebula has been convincingly detected by XMM-Newton, within a bubble-like structure $\sim 3.5 \mathrm{pc}$ in diameter (Güdel et al. 2008). The fast stellar wind from $\theta^{1}$ Ori is likely responsible for the bubble structure. Compared with NGC 6888, the bubble of $\theta^{1}$ Ori has a similar plasma temperature, $\sim 2 \times 10^{6} \mathrm{~K}$, but its X-ray luminosity, $\sim 5.5 \times 10^{31}$ erg $\mathrm{s}^{-1}$, is almost 3 orders magnitude lower than that of NGC 6888. This lower X-ray luminosity is caused by a weaker stellar wind and a lower ambient gas density.

The OB associations in the Rosette Nebula and the Omega Nebula (M17) are so young that no supernovae have occurred, and thus the hot gas in these two superbubbles has been powered solely by fast stellar winds. Chandra observations of the Rosette Nebula did reveal diffuse X-ray emission (Townsley et al. 2003), but a deep subsequent observation resolved more point sources from the faint diffuse emission (Townsley, personal communication). Chandra observations of the Omega Nebula show a dominant component at $7 \times 10^{6} \mathrm{~K}$ and a secondary component at $1.5 \times 10^{6} \mathrm{~K}$. This high-temperature component is not commonly seen in diffuse X-ray emission powered by fast stellar winds, as shown in Table 1 . The presence of $7 \times 10^{6} \mathrm{~K}$ hot gas in the Omega Nebula may be attributed to colliding stellar winds among the $\mathrm{O}$ stars in the cluster and/or the strong magnetic field that hinders thermal conduction at its interface with the cool dense nebular material (Dunne et al. 2003).

$X M M-N e w t o n$ observations of the superbubble N51D (Figure 6, left panel) in the LMC H II complex N51 have been used in conjunction with optical observations of the ionized swept-up shell, radio H I 21-cm line observations of the neutral swept-up shell, and spectroscopic and photometric data of the underlying massive star population to examine the energy budget. It is found that the total energy retained in the thermal and kinetic
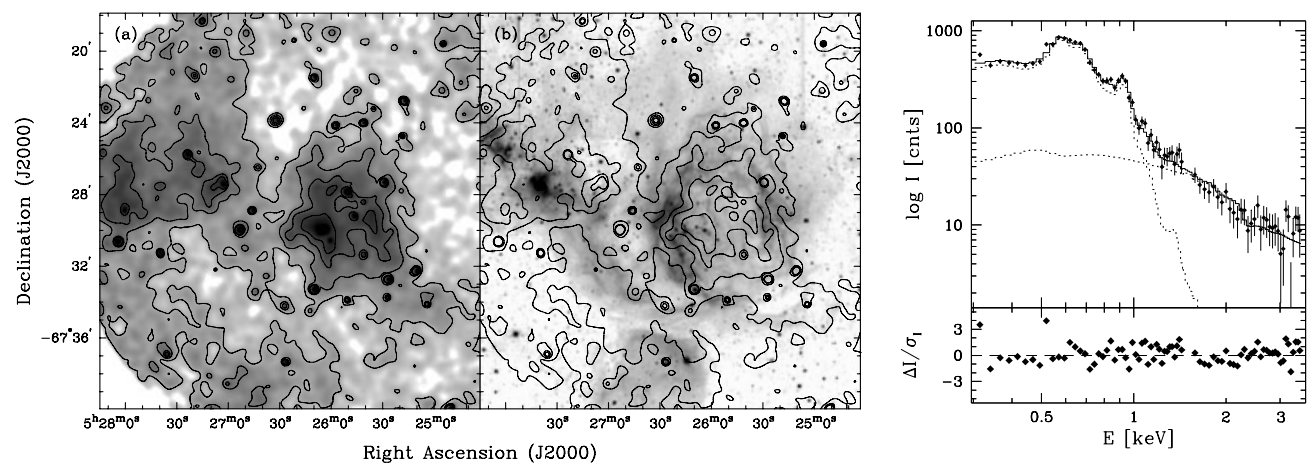

Figure 6. Left - XMM-Newton EPIC/pn X-ray and optical $\mathrm{H} \alpha$ images of the H II complex N51. The X-ray contours are over-plotted on the $\mathrm{H} \alpha$ image. Right - XMM-Newton EPIC/pn spectrum overlaid with the spectral components of the best-fit model. The residuals are plotted underneath the spectrum. These figures are taken from Cooper et al. (2004). 
energies in the superbubble N51D is only about $1 / 3$ of total stellar mechanical energy injected into the ambient medium (Cooper et al. 2004). It is possible that some energy has been used to accelerate particles that generate nonthermal X-ray emission (Figure 6, right panel). Similar energy budget problem is observed in the LMC superbubble 30 Dor C, which also exhibits nonthermal X-ray emission (Smith \& Wang 2004).

Nonthermal X-ray emission is perhaps the most unexpected discovery from Chandra and XMM-Newton observations of superbubbles: RCW 38 (Wolk et al. 2002), 30 Dor C (Bamba et al. 2004; Smith \& Wang 2004), and N51D (Cooper et al. 2004). Recent Suzaku observations of the superbubble in the LMC HII complex N11 also detected nonthermal X-ray emission (Maddox et al. 2008). Nonthermal emission at X-ray wavelengths requires very energetic particles. Smith \& Wang (2004) considered synchrotron radiation and inverse Compton scattering of optical photons, but concluded that the origin of the nonthermal X-ray emission was uncertain. It is possible that energetic particles in superbubbles are generated by repeated shock acceleration due to the high supernova explosion rate (Parizot et al. 2004).

\subsection{Interface Layers}

At the interface between a cold swept-up shell and its hot interior, thermal conduction takes place through diffusion, resulting in mass evaporation from the cold dense shell into the hot interior. Evaporation or ablation of dense clumps left behind in a bubble interior also injects mass into the hot gas. Interface layers play a very important role in the thermal evolution of a bubble because its lower temperatures $\left(10^{5} \mathrm{~K}\right)$ and higher densities lead to higher cooling rates, which regulate the temperature of the hot interior.

Interface layers are traditionally observed through interstellar absorption lines of highly ionized species, e.g., CIV, N V, and O VI. Such observations, however, are made at the mercy of the availability of probe stars whose continua provide the backdrop of the absorption lines. Chu et al. (2004) compared FUSE observations of O VI $\lambda \lambda 1031,1037$

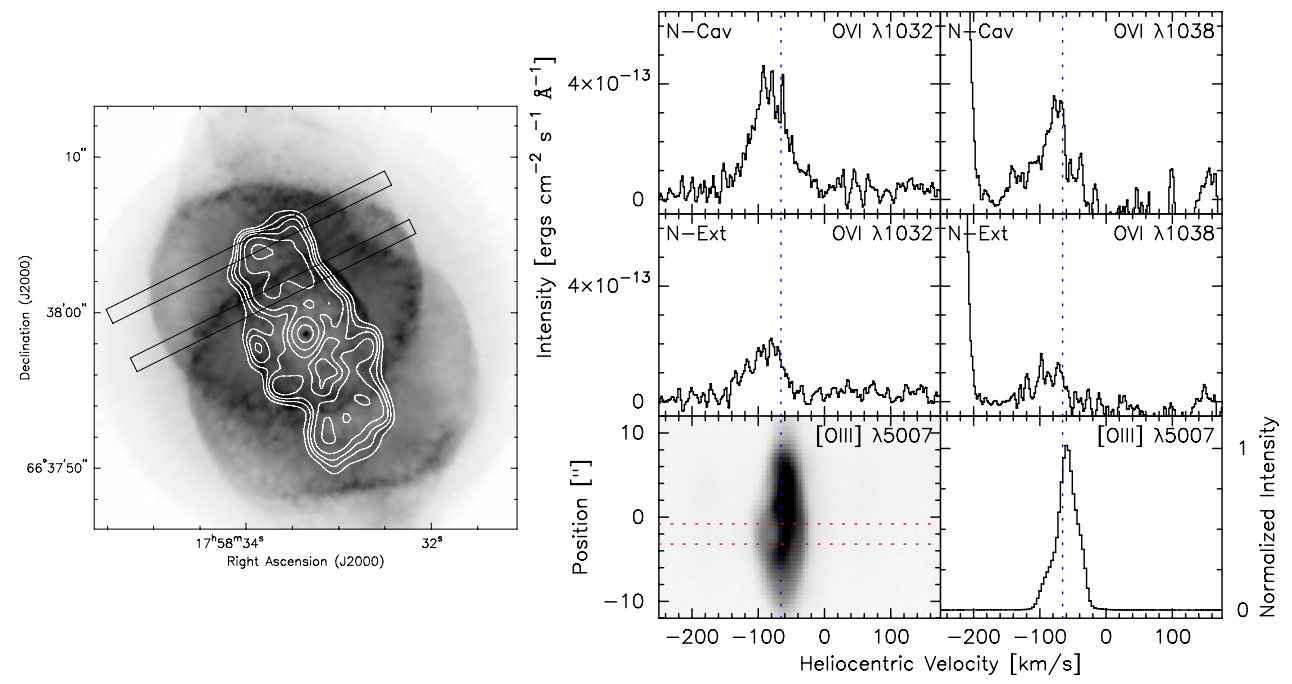

Figure 7. Left - HST H $\alpha$ image of NGC 6543 overlaid with X-ray contours extracted from a smoothed Chandra ACIS-S image. Locations of the FUSE HIRS apertures (N-Ext and N-Cav) are marked. Right - The O VI line profiles in the top four panels. The bottom left panel shows a EW-oriented long-slit echellogram centered at $4.5^{\prime \prime}$ north of the central star. The bottom right panel shows the [O III] velocity profile near the FUSE apertures. These figures are taken from Gruendl et al. (2004). 
lines in absorption and in emission for planetary nebulae, and suggested that conduction layers were easier to observe in emission rather than absorption. Figure 7 shows FUSE observations of two slit positions in the planetary nebula NGC 6543, clearly detecting the O VI emission from the interface layer (Gruendl et al. 2004).

NGC 6888 and S308 are the only two circumstellar bubbles detected in X-rays, but their central stars are too bright for FUSE observations of O VI absorption. Spatially resolved FUSE observations of O VI emission and HST STIS observations of the NV emission from S 308's interface layer would shed light on the conduction front or mixing layer; these observations were awarded but neither was carried out because of the premature demise of the instruments. Planetary nebulae are smaller versions of circumstellar bubbles. Diffuse X-ray emission from NGC 6543 has been detected; the limb-brightened morphology of the X-ray emission is expected from thermal conduction, but the X-ray luminosity is an order of magnitude lower than circumstellar bubble models with conduction (Chu et al. 2001). This raised doubt about thermal conduction. FUSE observations of the O VI emission from the conduction layer of NGC 6543 (Figure 7) were analyzed. Compared with circumstellar bubble models with thermal conduction, the O VI emission is an order of magnitude lower than expected; however, for the observed X-ray luminosity (an order or magnitude lower than expected) the O VI emission strength is roughly consistent with that expected from a conduction layer (Gruendl et al. 2004).

FUSE observations of O VI absorption from LMC superbubbles do not show obvious enhanced column density over the overwhelming LMC halo component (Howk et al. 2002). O VI emission from LMC superbubbles was serendipitously detected from archival FUSE observations, but it is not clear whether the emission arises from conduction interface, SNR shocks, or turbulent mixing layers (Sankrit \& Dixon 2007).

Besides FUSE, the SPEAR imaging spectrometer also provides useful O VI observation of superbubble. Kregenow et al. (2006) have presented SPEAR observations of the Eridanus superbubble and used the spatial distribution of O VI and C IV emission along the shell rim to illustrate that the emission originates from the thermal conduction layer.

The current lack of functioning high-dispersion UV spectroscopic observing facilities is worrisome; without such, it is difficult to advance our understandings of the interface layer. Bubble models tending details of thermal conduction at the interface (Arthur, in this volume) are needed to interpret UV and X-ray observations of bubbles.

\section{References}

Arthur, S. J., Dyson, J. E., \& Hartquist, T. W. 1993, MNRAS, 261, 425

Arthur, S. J., Henney, W. J., \& Dyson, J. E. 1996, A\&A A, 313, 897

Bamba, A., Ueno, M., Nakajima, H., \& Koyama, K. 2004, ApJ, 602, 257

Bochkarev, N. G. 1988, Nature, 332, 518

Book, L. G., Chu, Y.-H., \& Gruendl, R. A. 2008, ApJS, 175, 165

Boroson, B., Blair, W. P., Davidsen A. F. et al. 1997, ApJ, 478, 638

Braunsfurth, E., \& Feitzinger, J. V. 1983, A\&A, 127, 113

Burrows, D. N., Singh, K. P., Nousek, J. A., Garmire, G. P., \& Good, J. 1993, ApJ, 406, 97

Cappa, C. E., Arnal, E. M., Cichowolski, S., et al. 2003, in: K. A. van der Hucht, A. Herrero, \& C. Esteban (eds.), A Massive Star Odyssey: From Main Sequence to Supernova, (San Francisco: ASP), Proc. IAU Symp. 212, 596

Castor, J., McCray, R., \& Weaver, R. 1975, ApJ, 200, L107

Chen, C.-H. R., et al. 2008, submitted to ApJ

Chu, Y.-H. 2003, in: K. A. van der Hucht, A. Herrero, \& C. Esteban (eds.), A Massive Star Odyssey: From Main Sequence to Supernova, (San Francisco: ASP), Proc. IAU Symp. 212, 585 
Chu, Y.-H., Chang, H.-W., Su, Y.-L., \& Mac Low, M.-M. 1995, ApJ, 450, 157

Chu, Y.-H., Gruendl, R. A., \& Guerrero, M. A. 2004, in: M. Meixner, J. H. Kastner, B. Balick \& N. Soker (eds.), Asymmetrical Planetary Nebulae III: Winds, Structure and the Thunderbird, (San Francisco: ASP), ASP Conf. Series, 313, 254

Chu, Y.-H., Guerrero, M. A., Gruendl, R. A., 2001, ApJ, 553, L69

Chu, Y.-H., Guerrero, M. A., Gruendl, R. A., et al. 2003, ApJ, 599, 1189

Chu, Y. H., \& Lasker, B. M. 1980, PASP, 92, 730

Chu, Y.-H., \& Mac Low, M.-M. 1990, ApJ, 365, 510

Chu, Y.-H., Mac Low, M.-M., García-Segura, G. et al. 1993, ApJ, 414, 213

Chu, Y.-H., Wakker, B., Mac Low, M.-M. \& García-Segura, G. 1994, AJ, 108, 1696

Cooper, R. L., Guerrero, M. A., Chu, Y.-H., et al. 2004, ApJ, 605, 751

Dunne, B. C., Chu, Y.-H., Chen, C.-H. R. et al. 2003, ApJ, 590, 306

Dunne, B. C., Points, S. D., \& Chu, Y.-H. 2001, ApJS, 136, 119

Dyson, J. E., \& de Vries, J. 1972, A\&A, 20, 223

Ehlerová, S., \& Palouš, J. 2005, Ap\&AA, 437, 101

Freyer, T., Hensler, G., \& Yorke, H. W. 2003, ApJ, 594, 888

Freyer, T., Hensler, G., \& Yorke, H. W. 2006, ApJ, 638, 262

García-Segura, G., \& Mac Low, M.-M. 1995, ApJ, 455, 145

García-Segura, G., Langer, N., \& Mac Low, M.-M. 1996a, A\&A, 316, 133

García-Segura, G., Mac Low, M.-M., \& Langer, N. 1996b, A\&̋A, 305, 229

Goudis, C., \& Meaburn, J. 1978, A\&A A, 68, 189

Gruendl, R. A., Chu, Y.-H., \& Guerrero, M. A. 2004, ApJ, 617, L127

Gruendl, R. A., Guerrero, M. A., \& Chu, Y.-H. 2003, BAAS, 35, 746

Güdel, M., Briggs, K. R., Montmerle, T. et al. 2008, Science, 319, 309

Hatzidimitriou, D., Stanimirovic, S., Maragoudaki, F. et al. 2005, MNRAS, 360, 1171

Howarth, I. D. \& Phillips, A. P. 1986, MNRAS, 222, 809

Howk, J. C., Sembach, K. R., Savage, B. D. et al. 2002, ApJ, 569, 214

Jenkins, E. B. \& Meloy, D. A. 1974, ApJ, 193, L121

Kim, S., Dopita, M. A., Staveley-Smith, L., \& Bessell, M. S. 1999, AJ, 118, 2797

Kregenow, J., Edelstein, J., Korpela, E. J. et al. 2006, ApJ, 644, L167

Mac Low, M.-M. \& McCray, R. 1988, ApJ, 324, 776

Mac Low, M.-M., McCray, R., \& Norman, M. L. 1989, ApJ, 337, 141

Maddox, L. A., et al. 2008, ApJ submitted

Mathews, W. G. 1966, ApJ, 144, 206

Meaburn, J. 1980, MNRAS, 192, 365

Moffat, A. F. J. \& Robert, C. 1994, ApJ, 421, 310

Nazé, Y., Chu, Y.-H., Points, S. D. et al. 2001, AJ, 122, 921

Nazé, Y., Chu, Y.-H., Guerrero, M. A. et al. 2002, AJ, 124, 3325

Nichols-Bohlin, J. \& Fesen, R. A. 1993, AJ, 105, 672

Oey, M. S. 1996, ApJ, 467, 666

Oey, M. S. \& Clarke, C. J. 1997, MNRAS, 289, 570

Oey, M. S. \& García-Segura, G. 2004, ApJ, 613, 302

Oey, M. S. \& Smedley, S. A. 1998, AJ, 116, 1263

Parizot, E., Marcowith, A., van der Swaluw, E., et al. 2004, Ap\& $A, 424,747$

Pikel'Ner, S. B. 1968, ApL, 2, 97

Pikel'Ner, S. B. \& Shcheglov, P. V. 1969, Soviet Astronomy, 12, 757

Pittard, J. M., Dyson, J. E., \& Hartquist, T. W. 2001a, A\&A, 367, 1000

Pittard, J. M., Hartquist, T. W., \& Dyson, J. E. 2001b, A\&AA, 373, 1043

Sankrit, R. \& Dixon, W. V. D. 2007, PASP, 119, 284

Smith, D. A. \& Wang, Q. D. 2004, ApJ, 611, 881

Staveley-Smith, L., Sault, R. J., Hatzidimitriou, D. et al. 1997, MNRAS, 289, 225

Steigman, G., Strittmatter, P. A., \& Williams, R. E. 1975, ApJ, 198, 575

Strickland, D. K. \& Stevens, I. R. 1998, MNRAS, 297, 747

Tomisaka, K. 1992, PASJ, 44, 177 
Townsley, L. K., Feigelson, E. D., Montmerle, T. et al. 2003, ApJ, 593, 874

Weaver, R., McCray, R., Castor, J. et al. 1977, ApJ, 218, 377

Wolk, S. J., Bourke, T. L., Smith, R. K. et al. 2002, ApJ, 580, L161

Wrigge, M. 1999, Ap\&AA, 343, 599

Wrigge, M., Wendker, H. J., \& Wisotzki, L. 1994, Ap\& $\mathcal{E}$, 286, 219

\section{Discussion}

MORRIS: We've managed to obtain Spitzer/IRS spectra over some regions of the S 308 nebula, including what you identify as the thermal conduction zone. We don't see [Ne V] however. Is this a serious problem to constrain the conditions or even the existence of the thermal conduction zone?

CHu: The "conduction zone" speculated by Chu et al. (2003) is really the unperturbed RSG wind layer, as we have found from follow-up echelle observations of the shell rim (see Figure 5). The conduction zone would be immediately adjacent to the $\mathrm{X}$-ray emission edge. Boroson et al. (1997) detected C IV and N V absorption toward the central star, and demonstrated convincingly the existence of conduction layer in S 308. Previously, Shull (1974, ApJ, 212, 102) detected O VI absorption, which also unambiguously demonstrates the existence of conduction layer. Recently, Freyer et al. (2006) suggest that the soft X-ray emission originates from the swept-up RSG wind, then the conduction zone would be closer in, but the nebular gas does not show any material expanding at $400 \mathrm{~km} \mathrm{~s}^{-1}$ expected from their model. More observations of the region near the X-ray emission front are needed to search for and locate the conduction zone.

OEY: Earlier in this meeting we heard of the importance of mass loss from near breakup rotation velocities. Can you comment on the effect of this kind of mass loss on the circumstellar media?

CHu: There are no observations that indicate a link between the stellar rotation velocities and the physical properties of circumstellar nebulae. The aspherical geometry of a circumstellar nebula may be caused by rapid stellar rotation, but the effect of a close binary companion may also be very important.

VANBEVEREN: I noticed that the table where you list the WR stars and their bubbles only contains WR single stars. Are there WR+OB binaries with bubbles and, if not, is there a reason why only WR single stars have these bubbles?

CHu: As far as I know, the majority of WR stars in bubbles are single WN stars. One bubble (DEM L39) has a WC central star and another (G2.4+1.4) has a WO central star. The central star of DEM L231 (N57C shown in Figure 1) has a spectral type of WN4+B, the only WR binary in a nice bubble. More single than binary WR stars have bubbles. I would guess that colliding winds in a close binary system breaks the spherical symmetry and makes it difficult to form bubbles. 


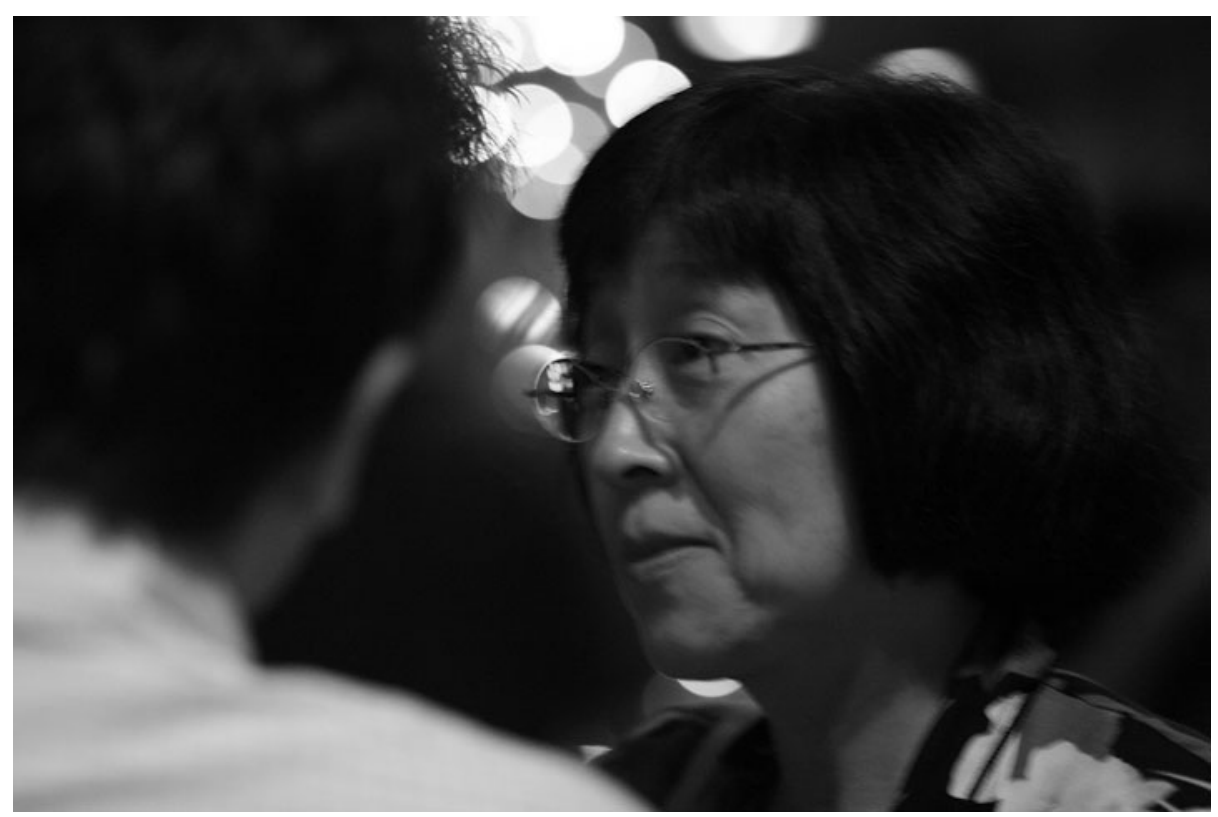

You-Hua Chu.

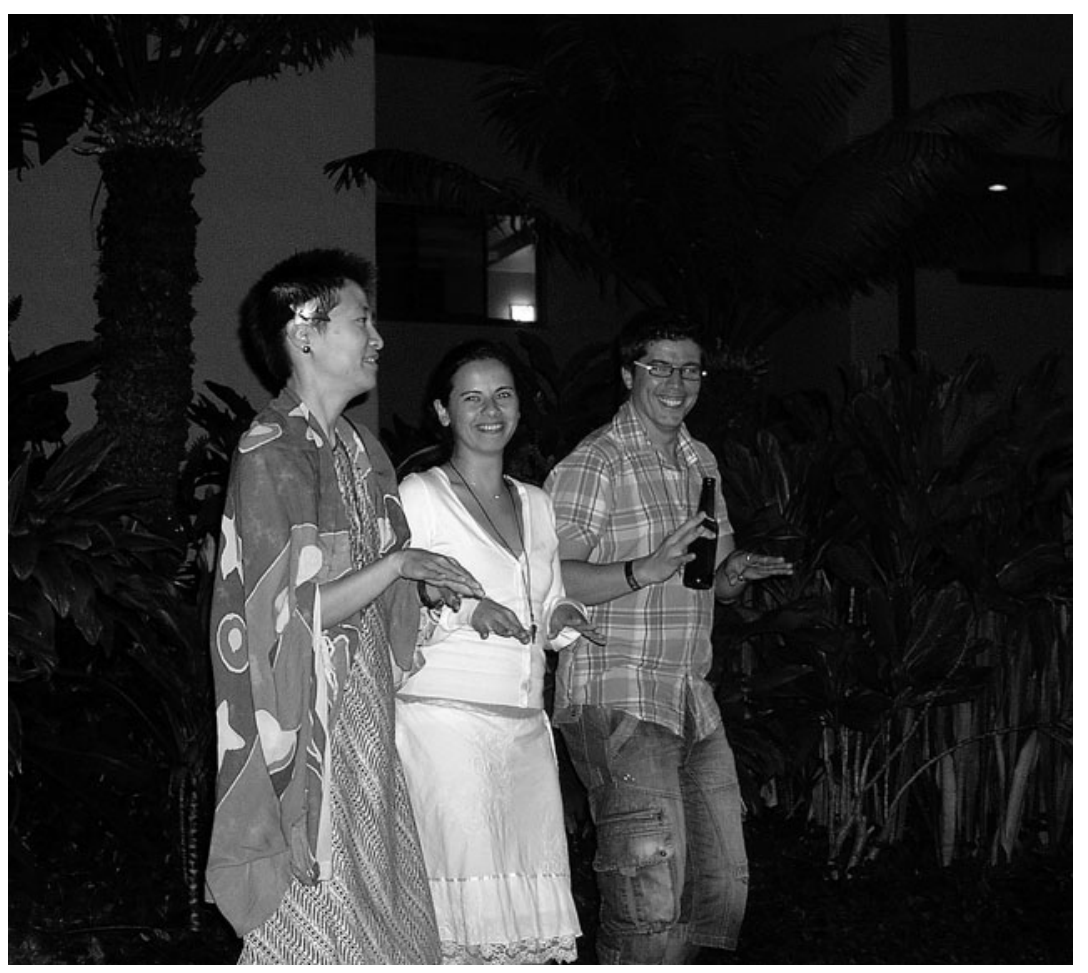

Sally Oey, Maria Nieva and Sergio Simón-Díaz. 